\title{
Analysis on the Detection Technology of Illegal Addition of Chemicals to Health Products
}

\author{
Ting Yang ${ }^{1}$, Qing Song ${ }^{2, *}$ \\ ${ }^{1}$ Kunming Medical University Haiyuan College, Kunming, Yunnan, 650106 \\ ${ }^{2}$ Yunnan Center for Disease Control and Prevention, Kunming, Yunnan, 650022 \\ *Corresponding author: Qing Song, E-mail: 707762264@qq.com
}

Keywords: Health Products, Illegal Addition, Detection Technology

\begin{abstract}
Health foods are foods that are suitable for consumption by a specific population and that have a regulating effect and are not intended to treat diseases. This paper systematically sorts out, analyzes and summarizes the status, safety and specific detection technology of illegal additives in health foods in China in recent years, and provides reference for standardizing health food technology review and exploring fast and effective detection methods.
\end{abstract}

\section{Introduction}

Judging from the current situation, China's health food safety issues are mainly reflected in antibacterial drug pollution, organic matter pollution, heavy metal residues and illegal addition. Among them, illegal addition is the most serious problem. In recent years, although many laws and regulations on the regulation of health food quality have been introduced in China, there are few regulations on the detection of illegally added drugs in health foods. Therefore, laws and regulations on the detection of illegally added drugs in health foods should be further improved. Great research on detection technology provides protection for consumers' health.

\section{Status of illegal addition of chemicals to health foods}

According to the monitoring of health food products and literature research in recent years, the illegal chemical drugs present in health foods have the following statuses: 1) The source of the additives is unknown, and the chemical substances that may be added include: a. Prescription drugs, after the substances are added, they can not control the dosage in use, which can easily lead to adverse reactions, and even damage the liver and kidney functions of consumers. For example, adding ephedrine to weight-loss products can excite the central nervous system and improve the body. The speed of metabolism, which promotes weight loss, and it is also one of the raw materials for making ice, which is a national controlled drug. Long-term use can affect heart rate and even harm life; b. Structural analogs of existing drugs. These compounds are slightly modified on the basis of existing drug structures. The basic skeleton of the structure is similar, and there may be similar clinical effects. Since most structural analogs are not preclinical and clinical studies, there are Larger safety hazards, as reported in many literatures, the structural analogues of the type 5 phosphodiesterase inhibitor (PDE-5) detected in health foods belong to this type of addition; c. Drugs that have been withdrawn, such as fenfluramine, sibutramine, and amfepramone, have been popular for significant weight loss, but they cause cardiovascular and central nervous system adverse reactions, so US food and drug The FDA, the European Union, and the State Food and Drug Administration (SFDA) have recalled these drugs, but according to literature research and health food risk assessment reports, the detection rate of sibutramine in many weight loss health foods Still very high; d. Add new drugs or lead compounds that have not been approved; e. In the chemical synthesis of drugs, in order to reduce costs, some of the added pharmaceutical ingredients are added in the form of crude raw materials, and their impurities and possible potential risk substances are unknown. 
The dose of illegally added drugs is arbitrary. For example, the detection amount of different batches of caffeine in certain types of weight loss health foods is between 4 and $327 \mathrm{mg}$. Meanwhile, in order to evade inspection, some manufacturers have illegal products in some batches. Added, some do not exist illegally added.

The compatibility of illegally added drugs is not clear. There may be interactions between illegally added drugs in health foods and health food formulations, and the added drugs may also interact with drugs that consumers are taking, these are threatening the health of consumers.

\section{Illegal drug detection technology in health food}

The HPLC method is generally used in the quantitative examination of illegally added drugs in health foods. In the actual application of the technical method, the spectrum of the control is first drawn, the standard library is constructed, and then the sample retention time is compared with the database to initially screen out Abnormal chemical substances, and finally compare the chromatographic peaks of abnormal chemical substances, DNA spectra and DNA spectra of the control in the database, accurately and efficiently check for illegal additives, and must also perform LC-MS verification after final confirmation of illegal components. The method is more suitable for the detection of illegally added drugs in weight-loss health foods. Some foreign researchers have verified the UPLC-DAD detection method of more than 30 compounds with weight loss efficacy, such as laxatives, stimulants and appetite suppressants, based on gradient elution. Phosphate buffer solution is needed as the mobile phase. Separation and detection of more than 30 compounds in about 15 minutes, qualitative analysis of compounds by UV absorption spectroscopy, finally detected 9 illegally added compounds such as sibutramine, caffeine and phenolphthalein, and caffeine and west the addition amount of $\mathrm{Bu} \mathrm{Qu}$ Ming and others has reached the medical level, which is extremely harmful to the health of consumers.

The operation of the TLC method in practical applications is relatively simple and efficient, and is the main method for preliminary screening of illegal drugs. The TCL method applied to the detection of illegally added drugs in health foods was discovered by scholars such as Shi Yaqin. The TLC method of adding chemical hypoglycemic agents and proprietary Chinese medicines in health foods was used, and ethanol and chloroform were used in the detection process. The developing agent and the color developing agent are cesium potassium iodide test solution. The TLC method accurately analyzes and detects chemical hypoglycemic agents such as gliclazide in health foods, and the detection limit is $0.1-0.3 \mathrm{mg} / \mathrm{g}$. The main advantage of this method is that the operation is simple, fast, flexible, and not susceptible to external interference.

The full name of infrared spectroscopy is "infrared spectrophotometry", which is abbreviated as IR in English. From the nature, it belongs to molecular absorption spectroscopy. This technology can record the infrared spectrum of a large amount of materials into a computer to construct a complete infrared spectrum standard resource. The library, in practical applications, only needs to measure the infrared spectrum of the suspect component, and then compare with the spectrum in the standard resource library, can resolve the atomic group structure of the substance in a short time, and then determine which compound belongs and whether it is illegal. Add ingredients. Infrared spectroscopy plays a very important role in the analysis of sugar sequence type. At the same time, this method is more suitable for the detection of illegally added drugs in chitin health foods. The short-term infrared spectroscopy technology is abbreviated as NIR. This technology has high efficiency and simple operation. It has been widely promoted and applied in the field of drug detection and analysis. For the illegal drug detection in health food, this technology is mainly suitable for qualitative detection. Deviations are easy to occur in the test.

Enzyme-linked immunosorbent assay (ELISA) is mainly based on the antigen-antibody immune response, and the enzyme is applied to the color reaction and identified by fluorescence reaction. China has issued relevant documents to use xanthan gum as a limited amount of added substances in food. Since xanthan gum can greatly improve the viscosity of foods and easily form colloids, many illegal food enterprises add a large amount of yellow in health foods for their own personal benefit. Raw rubber. Qin Hongmei and other scholars have confirmed that the enzyme-linked 
immunosorbent assay can accurately detect the hydrosol components such as xanthan gum present in health foods, and the method has the advantages of low cost and convenience, and can effectively reduce foods. The influence of some interfering substances on the detection accuracy.

LC-MS $\mathrm{Li}$ et al established a liquid chromatography-electrospray ionization tandem mass spectrometry (LC-ESI-MS/MS) method with clenbuterol as an internal standard to simultaneously determine the illegal addition of pseudoephedrine in diet-reducing foods. There are 7 illicit drugs for pseudoephedrine, caffeine, strychnine, fenfluramine, sildenafil and bupropion. $0 \%$. The mixture is separated by a RP - C18 column, with a concentration of $0.03 \%$. The acetonitrile of formic acid and a $0.1 \%$ aqueous formic acid solution were eluted in a gradient, and the 7 components were well separated within $10 \mathrm{~min}$. The recovery rate of the method was $81.2 \%$ to $110.3 \%$, and the detection limit was $4.2 \sim 16.7 \mu \mathrm{g} \bullet \mathrm{kg}-1$, and the relative standard deviation was $1.1 \% \sim 8$. 4\%. Ma Wei et al established accelerated consumption of fenfluramine, phenylpropanolamine, sibutramine, sertraline, rimonabant, bupropion, and west by accelerated solvent extraction (ASE). LC-MS/MS analysis of 11 appetite suppressants of citram, fluoxetine, fenfluramine, topiramate, and zonisamide. ESI ionization, MRM mode detection, retention time and particle ion characterization, external standard method for quantification. The detection limit of the method is 0.05 to $4.0 \mathrm{mg} \bullet \mathrm{kg}-1$, and the recovery rate is $78.3 \%$ to $103.6 \%$. The relative standard deviation is less than $15 \%$. At the same time, weight loss coffee, slimming tea, and weight loss are also established. LC-MS/MS analysis method for illegal addition of phenolphthalein and emodin in capsules, apple cider vinegar chewable tablets, slimming biscuits, etc. The detection rate of emodin was $25 \%$, and the detection rate of phenolphthalein was $66.7 \%$.

\section{Development trend of illegal drug detection technology in health food}

Despite the rapid development of China's health food safety testing technology in recent years, there is a large gap between China and foreign developed countries. With the variety of health foods and the increasing complexity of their constituents, the requirements for illegal drug detection technologies are becoming higher and higher. Therefore, it is necessary to further study the detection technology and appropriately introduce advanced extraction technologies, such as matrix solid phase. Disperse extraction and accelerated solvent extraction, etc., can reduce the amount of solvent used, achieve cost-saving purposes, and also play a better green environmental protection. Considering the complexity of the ingredients of health foods, it is necessary to purify the extracts, such as acidified silica gel column purification and solid phase extraction column purification. The purification method can greatly reduce the pollution probability of the detection instrument and save the cost of replacing the instrument. In addition, in response to the gradual increase in the content of permanent organic pollutants in current health foods, new technologies such as solid phase extraction combined with liquid chromatography and accelerated solution extraction combined with liquid chromatography can be introduced to detect illegally added drugs in health foods. The results are accurate and efficient. With the continuous development of the times, China has gradually focused on the illegal addition of drugs such as pesticide residues, organic pollutants and veterinary drug residues, which are extremely harmful to people's health, in the research of illegally added drug detection technology for health foods.

\section{Conclusion}

With the rapid improvement of production and living standards, people pay more attention to the prevention of diseases and the adjustment of sub-health status. Health foods will have greater development and market prospects, and a large number of varieties can be researched and developed. The safety of health foods and the establishment of a health food safety monitoring system are particularly important. Although the above methods are fast, simple, and highly sensitive, they are only suitable for laboratory testing, which cannot be done by the general consumer, and therefore are not universal. The author believes that not only should we strengthen international cooperation and exchange on the basis of existing tests, introduce internationally relevant testing 
technologies, and constantly improve the quality control and functional testing methods for health foods in China, making them better for China's health products. Testing service. It is also required that researchers invent more simple and easy-to-use testing methods or testing instruments so that consumers can test the products they buy at home, so that they can truly eat and rest assured that they will be illegally unknowingly unknowingly The harm of additives to health care products.

\section{References}

[1] Ding Shumin, Zhu Pinkia, Shi Yaqin, et al. Simultaneous determination of chlorogenic acid, cryptochlorogenic acid, caffeic acid and luteolin in rush grass by UPLC[J]. Journal of Chinese Medicinal Materials, 2013, 36(2): 246- 248.

[2] Wu Xiaoyong, Fu Xiaoying, Zhang Xiaodan, et al. HPLC-DAD-MS method for the detection of illegally added melatonin in traditional Chinese medicine preparations[J]. Chinese Journal of Pharmaceutical Sciences, 2011, 27(2): 153-155.

[3] Ma Wei, Peng Tao, Zhu Mingda, et al. Simultaneous Determination of 11 Appetite Inhibitors in Weight Loss Health Foods by Accelerated Solvent Extraction-High Performance Liquid Chromatography-Tandem Mass Spectrometry[J]. Chinese Journal of Analytical Chemistry, 2009, 37(11):1583-1589.

[4] Zhu Mingda, Chen Dongdong, Ma Wei, et al. Simultaneous Determination of Seven Insulin Secretion Promoters in Traditional Chinese Medicines and Health Foods by Dispersive Solid Phase Extraction and High Performance Liquid Chromatography-Tandem Mass Spectrometry[J]. Chinese Journal of Analytical Chemistry, 2011, 39(2): 213-218.

[5] Li Lanzhen, Wang Wei. Research progress on detection technology of illegal food additives in health foods [J]. Dietary Health, 2016, 3(1): 200. 